\title{
articles
}

\section{Predicting frequency of suicide attempts of adolescent outpatients at Weskoppies Hospital using clinical and demographic characteristics}

\author{
G Fine, $M B B C h$ \\ H C Alison, MB BCh, MMed (Psych), FCPsych (SA), Cert Child \\ Psych (SA) \\ D van der Westhuizen, MB ChB, MMed (Psych), FCPsych (SA), \\ $\mathrm{MD}(\mathrm{SA})$, Cert Child Psych (SA) \\ C Krüger, MB BCh, MMed (Psych), FCPsych (SA), MD (Warwick, UK) \\ Department of Psychiatry, University of Pretoria and Weskoppies \\ Hospital, Pretoria
}

The prevention of suicide, particularly adolescent suicide, remains one of the biggest challenges in psychiatry.

Objectives. To ascertain: (i) clinical and demographic characteristics; and (ii) possible associations between these characteristics and suicide attempt frequency in a selected patient group at Weskoppies Hospital over 4 months.

Methods. Fifty adolescent outpatients aged between 13 and 17 years with a history of one or more suicide attempts were interviewed to obtain demographic and clinical features. Chisquare and Fisher's exact tests assessed associations between these features and suicide attempt frequency.

Results. Of the subjects, 79\% were aged between 15 and 17 years; they were predominantly female (62\%) and Caucasian (83\%). Mainstream and special education schools were equally represented. Three-quarters had reached grades $8-10$, and 14\% lived with both biological parents, 33\% in places of safety and $37 \%$ with one divorced parent. The minority of caregivers had a history of alcohol abuse and other substance use.

Twenty-nine per cent of the subjects had attempted suicide on more than 10 occasions and $23 \%$ had made a single attempt. The most common methods were wounding (74\%), tablet overdose (34\%) and hanging (20\%). Psychiatric diagnoses included major depressive disorder (64\%), bipolar disorder (38\%), alcohol abuse (18\%) and other substance abuse (24\%). Familial features included depression, substance abuse, antisocial behaviour and suicide. Familial suicidal behaviour included suicide attempts by parents (85\%), siblings (36\%), aunts and uncles (31\%) and cousins (44\%). Physical and sexual abuse was reported in $52 \%$ of families.

Conclusion. Many findings and profiles of other studies were confirmed and point to school and home environments, family psychopathology and psychiatric diagnoses as factors associated with adolescent suicide attempts. Associations between the frequency of suicide attempts and the demographic and clinical characteristics were statistically inconclusive.
Suicidal thinking and behaviour are of grave concern to the psychiatric community, with the prevalence of suicide attempts taking on alarming proportions in some countries. Several international studies have examined the lifetime prevalence of suicide attempts by adolescents between 13 and 18 years old, with figures of $2.7 \%$ in the USA, ${ }^{1} 3 \%$ in Norway ${ }^{2}$ and $6.5 \%$ in the USA and Germany, ${ }^{3}$ and an increase from $7 \%$ to $13.4 \%$ in Greece, ${ }^{4}$ being reported over the past few decades. The 12-month prevalence of suicide attempts by high-school students in a similar age range has been reported variously as $1.4 \%$ in the USA, $1.7 \%$ in Norway, $4.3 \%$ in a community sample in Australia and 12\% in Nigeria., 1,25,6

South African studies have also found high figures for the lifetime prevalence of attempted suicide in secondary school students. For example, Flisher et al. (2006) found that 18.6\% of female high school students had attempted suicide, and Cherian et al. (2000) that the frequency of attempted suicide was $11.3 \%$ among blacks, 13\% among whites and $13.5 \%$ among Asians in a group of secondary school pupils aged $17-24$ years.

Research and practice illustrates that suicidality is largely determined by two sets of characteristics, clinical and demographic. While some features are present among both adults and adolescents, the profile of adolescent suicide attempters differs from that of adults in certain crucial aspects.

Clinically, a strong correlation exists between certain psychiatric disorders and suicide attempts, particularly among adolescents. Numerous studies show that anorexia nervosa and body image, $^{9-12}$ health risk behaviours including sexual intercourse, damaging property and substance abuse, ${ }^{10-18}$ major depressive disorders, ${ }^{11-20}$ anxiety disorders, particularly panic disorder and post-traumatic stress disorder, ${ }^{11,12,16,20}$ impulse control disorders, ${ }^{20}$ externalising behaviours ${ }^{12,18}$ and other psychopathology, especially when linked with high environmental stress levels with a recent stressor including a recent romantic breakup, assault or arrest, contributed to the prediction of suicide attempts in multivariate analyses. ${ }^{12,18}$

In one survey of adolescents, 5\% reported over one suicide attempt per year. ${ }^{18}$ The factors leading to repeated attempts included depression (13 times increase), sexual assault (7 times increase), substance abuse disorder, violent behaviour in boys, and problems of increased weight in girls. ${ }^{18}$ In a 2001 Centers for Disease Control and Prevention Youth Risk Behavioral Survey of 13601 adolescents in school (grades 9 - 12), 53\% of those who had attempted suicide did so only once per year. ${ }^{18}$ One attempt increased the risk for further attempts 15 -fold, however; $30 \%$ had two or three attempts per year, and $17 \%$ four or more attempts in a year. ${ }^{18}$ 
Physically and sexually abused children have high rates of suicidal behaviour. ${ }^{19}$ Child abuse victims have a high incidence of suicidality, which may be directly attributable to the abuse or a consequence of the range of psychiatric problems associated with abuse. It is difficult for physically abused children to develop relationships, resulting in social isolation or conflictual relationships that predispose them to suicidal behaviour. Children with family members who completed suicide are at a higher risk of attempting suicide than children without such family members. ${ }^{19}$

Parental psychopathology is associated with adolescent suicidal behaviour. It remains unclear whether a family history of depression and substance abuse increases completed suicide risk. The purported association between dysfunctional families and adolescent suicide attempts is also unclear. ${ }^{20}$

Demographically, correlations have been found between adverse childhood experiences and suicide ideation and attempts. ${ }^{21}$ Gender- and age-based patterns of differentiation in methods and prevalence of suicide attempts, as well as differences across types and levels of education and race/ethnicity, have been researched.22

Male children are more likely than females to complete suicide. ${ }^{19}$ Girls are significantly more likely than boys to seriously consider attempting suicide, make a specific plan and attempt suicide. ${ }^{19}$

Age has also been presented as a key demographic factor in attempted suicide across many surveys. ${ }^{16,19}$ Rates of completed and attempted suicide are rare before puberty and increase during adolescence, increasing significantly in late adolescence and continuing to rise until the early twenties. This may be related to increases in co-morbid mood and substance use disorders. Suicide attempts peak in the late adolescent population, between the ages of 16 and 18 years, with a marked decrease in suicide attempts thereafter, especially for women. ${ }^{19}$

Studies have also pointed to national and cultural differences in the method of suicide and suicide attempts among youth. Investigations have moreover revealed that there does not seem to be a direct association between method choice and psychopathology. Choice of method is in fact largely determined through opportunity, availability and accessibility of tools, and local customs. ${ }^{19}$

Adolescent suicidality has tended to be researched in the setting of the developed world. South African-based studies that investigated adolescents showed that suicidal ideation/attempts were associated with the following: low levels of family connection and regulation, ${ }^{23}$ increased levels of conflict and psychological control in the parent-child relationship, ${ }^{23}$ depression and low self-esteem in the family context, ${ }^{24}$ low parental monitoring, ${ }_{1}^{25}$ and risk behaviours, e.g. smoking. ${ }^{25}$ Maternal closeness related to female adolescents was protective in reducing risk behaviours. ${ }^{26}$ Male gender and city of residence were associated with specific methods of suicide. ${ }^{27}$
No such research has been undertaken at Weskoppies Hospital. It was therefore considered worth examining the specific situation at this particular specialist psychiatric hospital.

This study was undertaken to ascertain whether the patterns described above would be replicated at Weskoppies Hospital, through profiling adolescent psychiatric patients attending the outpatient clinic who had a history of attempted suicide. This was done through the mapping of their clinical and demographic characteristics as potential predictors of the frequency of suicide attempts. If an association could be identified between these clinical and demographic characteristics and the frequency of suicide attempts, we hoped that it would assist in service delivery planning aimed at preventing such attempts.

More specifically the study aimed:

- to ascertain the demographic and clinical features of adolescents who had attempted suicide and characteristics of suicide attempts

- to assess the association between these clinical and demographic characteristics and frequency of suicide attempts.

\section{Method}

The research population for this study comprised all adolescents attending the outpatient clinic at Weskoppies Hospital over a 4-month period. The patients were screened during their outpatient appointments by the clinic registrars and psychiatrists and identified for participation on the basis of a history of a previous suicide attempt. On completion of the consultations, the potential participants were referred to the researcher, where informed assent and consent from the adolescent and parent/guardian were obtained respectively.

This study focused on both the clinical and demographic characteristics of the studied population, which often acted in combination. This required an investigation into the gender, age and race of the suicide attempters, as well as the type of school they attended and their living conditions. The methods used in the attempts, the prevalence of physical and sexual abuse, previous psychiatric disorder and parental psychopathology, and any family history of suicidal behaviour and abuse were also considered.

The researcher collected data from outpatients who met the following inclusion criteria:

- outpatients aged between 13 and 17 years

- adolescents with a previous history of suicide attempts who attended the Weskoppies outpatient clinic between December 2009 and March 2010.

The research returned 50 clinical cases to form the study group, as per the minimum sample requirements.

This study was a cross-sectional survey. Utilising a quantitative research design, the questionnaire encompassed a set of closed- 
ended questions that addressed the demographic and clinical features. The study relied primarily on collecting data through interviewing the relevant adolescents, and in some instances the caregivers when available.

The questionnaire required answers to the following demographic information: age, race, gender, schooling and living conditions.

The clinical data requested in the questionnaire included the following: methods of suicide attempts, personal history of psychiatric disorder, personal history of substance abuse, familial psychopathology, family history of substance abuse, family history of suicidal behaviour, and physical and sexual abuse of the respondent.

The project was registered with the Department of Statistics at the University of Pretoria, and once the required number of questionnaires was completed, the relevant information was captured electronically in a database. Contingency tables were then generated from the electronic data, from which findings were derived and analysed. Measurement was on the basis of a system of scaling, and the assignment of numerical codes to the answer options provided to the questionnaire.

The relationship between frequency of suicide attempt on the one hand, and demographic and clinical characteristics on the other, was analysed statistically by testing the difference between low-frequency suicide attempters and high-frequency suicide attempters, with respect to a number of demographic and clinical variables. The frequency of suicide attempts was categorised into three groups, i.e. one, two to five, or more than five attempts. For these comparisons, two-way tables and chi-square and Fisher's exact tests were used. Cases with missing data were excluded from analyses. Statistical analysis was done by the Department of Statistics, University of Pretoria.

The anonymity and confidentiality of participants were ensured throughout the study. An informed consent/assent form was devised and signed by the participants. The study was approved by the Research Ethics Committee of the Faculty of Health Sciences, University of Pretoria.

\section{Results}

\section{Demographic characteristics}

Age. Of the sample, most were over the age of 15; $79 \%$ of all respondents were aged between 15 and 17, with 16-year-olds accounting for the highest percentage (41\%), and 15-year-olds comprising a quarter of respondents.

Gender. The study revealed a preponderance of females in the target population, $62 \%$ of respondents being female and $38 \%$ male.

Race. Of the sample $83 \%$ were white and $17 \%$ black. There were no coloured or asian respondents.
Schooling. Most of the participants were currently in grade 8 (25\%), 9 (30\%) or 10 (19\%). The study revealed an equal representation of learners from mainstream schools (50\%) and the special educational sector (50\%)

Living conditions. Of the respondents, 33\% lived in a place of safety, $37 \%$ were residing with a divorced parent, $15 \%$ were in foster care, and only $14 \%$ lived with both their biological parents.

Substance abuse and history of caregivers. Of the adolescents interviewed, $17 \%$ indicated that their caregiver(s) were currently abusing alcohol, and $28 \%$ that their caregiver(s) had a previous history of alcohol abuse; $6 \%$ and $8 \%$ of caregivers, respectively, had a current or previous drug abuse history.

\section{Clinical characteristics}

Frequency of suicide attempts. Almost a third of the respondents (29\%) had attempted suicide in excess of 10 times, $23 \%$ had made a single attempt, and $22 \%$ reported 2 - 5 attempts (22\%).

Most recent suicide attempt. There was an equal distribution of respondents whose most recent suicide attempt had been 1 month, 2 - 6 months and more than 6 months prior to the interview.

Methods of suicide attempt. In outlining the most widely utilised method of suicide, the study found that almost three-quarters of all the respondents had engaged in a practice of wounding (cutting or self-mutilation). Wounding may not necessarily represent a suicide attempt, but in adolescence the intent underlying highrisk behaviour like wounding can be suicidal in nature. The intent is often difficult to distinguish. For this reason, wounding was included as a form of suicide attempt. In addition to wounding, the most widely utilised methods among the interviewed group included an overdose of tablets (34\%), hanging (20\%), jumping from a height (6\%), drowning (4\%) and poisoning (4\%).

Diagnosis. The most common psychiatric diagnoses among the respondents were major depressive disorder (64\%), conduct disorder (46\%), attention deficit hyperactivity disorder (40\%) and bipolar disorder (38\%), while rates of alcohol and drug abuse were $18 \%$ and $24 \%$, respectively. Eating disorders and anxiety disorders accounted for $2 \%$ each.

Familial psychopathology. There was a high incidence of family psychopathology, including depression (57\%), substance abuse (43\%), antisocial behaviour (36\%) and physical and/or sexual abuse (42\%). It was also found that the families of the respondents had high prevalences of suicidality, with $85 \%$ of parents, $36 \%$ of siblings, $44 \%$ of cousins and $31 \%$ of uncles and/or aunts having engaged in suicidal behaviour.

Abuse. A history of physical and/or sexual abuse was mentioned by a small majority (52\%) of respondents. 


\section{Demographic and clinical characteristics and frequency of suicide attempts}

Chi-square test. The chi-square test proved invalid because there was a larger than acceptable proportion of 'Did not know' answers, revealing more than the standard number of cells with expected counts less than 5 . A small sample size also contributed to the chisquare test being invalid.

Fisher's exact test. The results of the subsequent Fisher's exact test examining the associations between the set of individual demographic and clinical characteristics mentioned above and the frequency of suicide attempts are set out in Table 1.

\section{Discussion}

This two-part investigation into the demographic and clinical profile of a sample of 50 adolescent outpatients with a history of suicide attempts at Weskoppies Hospital, Pretoria, provides at least a baseline indication of the major features and characteristics of this patient group.

The second part of the assessment, concerning prediction of the frequency of suicide attempts through these demographic and clinical characteristics, proved at best statistically inconclusive. With respect to the attempt at discerning the nature and extent of the association between the demographic and clinical characteristics and the frequency of suicide, and with due recognition of the invalidity of the chi-square test, it was observed that the Fisher's exact tests were all statistically insignificant.

While taking into account certain limitations, some conclusions can be drawn. The respondents were mainly white, mainly female, in age-appropriate grades at school, and with a higher proportion of attendance at special education schools than the general population. It is also striking that the vast majority of the respondents did not live in a two-parent household, rather being concentrated in places of safety, foster homes and divorced oneparent households. While it cannot be definitively concluded from the current study that there is direct causality between such school

\begin{tabular}{|c|c|}
\hline Demographic and clinical variable & $p$-values* \\
\hline $\begin{array}{l}\text { Living conditions against frequency of suicide } \\
\text { attempts }\end{array}$ & 0.17 \\
\hline Physical abuse against frequency of suicide attempts & 0.18 \\
\hline Sexual abuse & 0.37 \\
\hline $\begin{array}{l}\text { Family history of suicidal behaviour against frequency } \\
\text { of suicide attempts }\end{array}$ & 0.85 \\
\hline $\begin{array}{l}\text { Alcohol and drug abuse by adolescents against } \\
\text { frequency of suicide attempts }\end{array}$ & 1 \\
\hline
\end{tabular}

and home environments and suicide attempts, these findings do seem to correspond to the preponderance of relatively unstable school and home environments among adolescent suicide attempters reported in the psychiatric literature cited above.

The study sample also exhibited patterns of psychiatric disorders akin to other reported studies, as well as a similar incidence of physical and sexual abuse among the adolescent suicide attempters. The precise patterns of cause and effect could not be clearly established.

The familial psychopathology of the suicide attempters interviewed also pointed to family histories of psychiatric disorders, substance abuse, antisocial behaviour, suicidal behaviour and completed suicides. These features have often also been apparent among adolescent suicide attempters in other studies.

While the study did arrive at valuable conclusions, the research also experienced a few challenges and limitations. In addition to the invalidity of the chi-square test described above, and its resolution through the utilisation of Fisher's exact test, we also did not utilise a control group of patients with similar disorders, without a history of suicide attempts, to compare with the study sample.

With due recognition of the fact that frequency can be a poor indicator of seriousness of suicide attempts, a large proportion of the respondents were found to have made multiple attempts. A similar proportion had only attempted suicide once. The high percentage of multiple attempts could be because this study included cutting and self-mutilation under the general rubric of suicide attempts. Wounding was included as a form of suicide attempt as intent and lethality in adolescents do not necessarily correspond directly. Wounding may have high intent but low lethality.

The findings from this study could serve as a useful starting point for further and more detailed research into the topic. Such further studies could examine the association between the frequency of suicide attempts and interventions, e.g. the use of medication, psychotherapy, psycho-education and substance abuse rehabilitation.

Implications for clinical practice include paying attention to specific clinical and demographic characteristics when assessing the suicide risk of adolescents at Weskoppies Hospital. It is conceivable that these findings could be included as a part of a risk assessment tool.

\section{Conclusion}

The association between frequency of suicide attempts and the respondents' demographic and clinical characteristics was statistically inconclusive. The study confirmed many of the findings and profiles of other studies, and points in particular to stressors in the school and home environment, family psychopathology and psychiatric diagnoses as possible risk factors for adolescent suicide attempts. 
Acknowledgements. We acknowledge the assistance of the statisticians at the University of Pretoria, Mr P J van Staden and Ms J Somerville, as well as Mrs M A Mabena, Chief Executive Officer of Weskoppies Hospital, for facilitating the research.

\section{References}

1. Joe S, Baser RS, Neighbors HW, Caldwell CH, Jackson JS. 12-month and lifetime prevalence of suicide attempts among black adolescents in the National Survey of American Life. J Am Acad Child Adolesc Psychiatry 2009;48(3):271-282.

2. Larsson B, Sund AM. Prevalence, course, incidence, and 1-year prediction of deliberate self-harm and suicide attempts in early Norwegian school adolescents. Suicide Life Threat Behav 2008;38(2):152-65.

3. Plener PL, Libal G, Keller F, Fegert JM, Muehlenkamp JJ. An international comparison of adolescent non-suicidal self-injury (NSSI) and suicide attempts: Germany and the USA. Psychol Med 2009;39(9):1549-1558

4. Kokkevi A, Rotsika V, Arapaki A, Richardson C. Increasing self-reported suicide attempts by adolescents in Greece between 1984 and 2007. Social Psychiatry \& Psychiatric Epidemiology 2011;46(3):231-237.

5. Sawyer MG, Guidolin M, Schulz KL, McGinnes B, Zubrick SR, Baghurst PA. The mental health and wellbeing of adolescents on remand in Australia. Aust N Z J Psychiatry 2010;44(6):551-

6. Omigbodun O, Dogra N, Esan O, Adedokun B. Prevalence and correlates of suicidal behaviour among adolescents in southwest Nigeria. Int J Soc Psychiatry 2008;54(1):34-46.

7. Flisher $\mathrm{AJ}$, Ward $\mathrm{CL}$, Liang $\mathrm{H}$, et al. Injury-related behaviour among South African highschool students at six sites. S Afr Med J 2006;96(9):825-830

8. Cherian VI, Cherian L, Peltzer K. Cross-cultural attitudes towards suicide among South African secondary school pupils. East Afr Med J 2000;77(3):165-167.

9. Brausch AM, Muehlenkamp JJ. Body image and suicidal ideation in adolescents Body Image 2007;4(2):207-212

10. Foulon C, Guelfi JD, Kipman A, et al. Switching to the bingeing/purging subtype of anorexia nervosa is frequently associated with suicidal attempts. Journal of the Association of European Psychiatrists 2007;22(8):513-519.

11. Bulik CM, Thornton L, Pinheiro AP, et al. Suicide attempts in anorexia nervosa. Psychosom Med 2008;70(3):378-383.

12. Fordwood SR, Asarnow JR, Huizar DP, Reise SP. Suicide attempts among depressed adolescents in primary care. J Clin Child Adolesc Psychol 2007;36(3):392-404.

13. Houck CD, Hadley W, Lescano CM, Pugatch D, Brown LK. Suicide attempt and sexual risk behavior: relationship among adolescents. Arch Suicide Res 2008;12(1):39-49.

14. Afifi TO, Cox BJ, Katz LY. The associations between health risk behaviours and suicidal ideation and attempts in a nationally representative sample of young adolescents. Can J Psychiatry 2007;52(10):666-674

15. O'Connor RC. Personality and cognitive process: self-criticism and different types of rumination as predictors of suicidal ideation. Behav Res Ther 2008:46(3):392-401.

16. Nolen S, McReynolds LS, DeComo RE, John R, Keating JM, Wasserman GA. Lifetime suicide attempts in juvenile assessment center youth. Arch Suicide Res 2008;12(2):111-123.

17. Swahn MH, Bossarte RM. Gender, early alcohol use, and suicide ideation and attempts: findings from the 2005 youth risk behavior survey. J Adolesc Health 2007;41 (2):175-181.

18. Greydanus DE, Calles J Jr. Suicide in children and adolescents. Primary Care: Clinics in Office Practice 2007;34(2):259-273.

19. Steele MM, Doey T. Suicidal behaviour in children and adolescents. Can I Psychiatry 2007;52:21S-33S.

20. Nock MK, Borges G, Bromet EJ, et al. Cross-national prevalence and risk factors for suicidal ideation, plans and attempts. Br J Psychiatry 2008;192(2):98-105.

21. Afifi TO, Enns MW, Cox BJ, Asmundson GJ, Stein MB, Sareen J. Population attributable fractions of psychiatric disorders and suicide ideation and attempts associated with adverse childhood experience. Am J Public Health 2008;98(5):946-952.

22. Yasan A, Danis R, Tamam L, Ozmen S, Ozkan M. Socio-cultural features and sex profile of the individuals with serious suicide attempts in southeastern Turkey: a one-year survey. Suicide Life Threat Behav 2008;38(4):467-480.

23. Van Renen L, Wild LG. Family functioning and suicidal ideation/behaviour in adolescents: a pilot study. Journal of Child and Adolescent Mental Health 2008;20(2):111-121.

24. Wild LG, Flisher AJ, Lombard C. Suicidal ideation and attempts in adolescents: associations with depression and six domains of self-esteem. J Adolesc 2004;27(6):611-624

25. King RA, Schwab-Stone M, Flisher AJ. Psychosocial and risk behaviour correlates of youth suicide attempts and suicidal ideation. J Am Acad Child Adolesc Psychiatry 2001;40(7):837-

26. Gilreath TD, King G, Graham JW, Flisher AJ. Associations between maternal closeness suicidal ideation and risk behaviours in Cape Town. European Child and Adolescent Psychiatry 2009;18(3):174-179.

27. Burrows S, Laflamme L. Suicide among urban South African adolescents. Int J Adolesc Med Health 2008;20(4):519-528. 